\title{
PLANO INDIVIDUALIZADO DE TRANSIÇÃO: POSSIBILIDADES PARA A INSERÇÃO DE PESSOAS COM DEFICIÊNCIA INTELECTUAL NO MUNDO LABORAL
}

Annie Gomes Redig ${ }^{1}$

Vanessa C. S. Pinheiro ${ }^{2}$

\begin{abstract}
Resumo
O presente trabalho foi realizado com o apoio da Coordenação de Aperfeiçoamento de Pessoal de Nível Superior (CAPES) - Brasil -, Código de Financiamento 001, e é baseado em uma revisão da literatura específica da área. Sendo assim, pretende-se discutir a temática da transição para a vida pósescola, com ênfase nas atividades laborais, para jovens e adultos com deficiência intelectual, tema ainda pouco explorado nas produções cientificas brasileiras. É preciso, portanto, repensar o percurso formativo do aluno com deficiência intelectual, de forma a contemplar o desenvolvimento de habilidades para uma vida independente, auxiliando no processo de transição da escola para a vida adulta e para o trabalho. Acreditamos que com a elaboração de práticas pedagógicas individualizadas, atreladas e organizadas por documentos como o Plano de Ensino Individualizado (PEI) e Plano Individualizado de Transição (PIT), esse percurso formativo poderá ser construído de forma a possibilitar que a transição da escola para a vida independente aconteça de maneira estruturada e eficiente. O Plano Individualizado de Transição se configura como uma estratégia pedagógica para auxiliar na escolarização do aluno com deficiência, dialogando com os conteúdos acadêmicos, seus interesses e desenvolvimento de habilidades laborais. Esse documento é um instrumento norteador do trabalho do professor, que organiza a ação pedagógica e o atendimento educacional especializado, com o objetivo de auxiliar nessa transição. Nessa direção, conclui-se que a individualização do ensino, aliada à acessibilidade pedagógica, uma equipe capacitada e com um trabalho colaborativo, é possível que tais educandos trilhem seus caminhos nos mais diferentes níveis de ensino e vida independente.
\end{abstract}

Palavras-chave: Pessoas com deficiência intelectual. Vida independente. Mundo do trabalho. Plano Individualizado de Transição.

\begin{abstract}
This study was funded in part by the Higher Education Personnel Improvement Coordination - Brazil (CAPES) - Finance Code 001e is based on a literature review. Thus, it is intended to discuss the theme of transition to after-school life, with emphasis on work activities, for young and adults with intellectual disabilities, a topic still little explored in Brazilian scientific productions. Therefore, it is necessary to rethink the educational path of students with intellectual disabilities, in order to contemplate the development of skills for independent living, helping in the process of transition from

\footnotetext{
${ }^{1}$ Doutora, Prof ${ }^{\mathrm{a}}$. Adjunta da Faculdade de Educação da Universidade do Estado do Rio de Janeiro, Rio de Janeiro, RJ, Brasil, annieredig@yahoo.com.br.

${ }^{2}$ Mestranda do Programa de Pós-Graduação em Educação da Universidade do Estado do Rio de Janeiro, Rio de Janeiro, RJ, Brasil, vanessapinheiro1509@gmail.com.
}

Fed. Nac. das Apaes- Fenapaes $\mid$ Brasília/DF | v.1 | nº 9

P. $44-56$

jan./jun.2018 | ISSN 2237-4329| 
school to adult life and work. We believe that with the development of individualized pedagogical practices linked and organized by documents such as the Individualized Teaching Plan (PEI) and Individualized Transition Plan (PIT), this formative pathway can be constructed to enable the transition from school to life. independently happens in a structured and efficient manner. The Individualized Transition Plan is configured as a pedagogical strategy to assist in the schooling of students with disabilities by dialoguing with academic content, interests and development of work skills. This document is a guiding instrument of the teacher's work, which organizes the pedagogical action and the specialized educational attendance, with the purpose of helping this transition. In this sense, it is concluded that the individualization of teaching allied to pedagogical accessibility, a team capable and collaborative work, it is possible that these students walk their paths in different levels of teaching and independent life.

Keywords: People with intellectual disabilities. World of work. Inclusion. Individualized Transition Plan.

\section{INTRODUÇÃO}

Muito se discute sobre a inclusão escolar de pessoas com deficiência, mas quais são as perspectivas para a etapa pós-escola? Quando pensamos no momento da saída da instituição de ensino, é preciso compreender que essa próxima fase é marcada pela incerteza de possibilidades de continuidade da escolarização e colocação no mercado de trabalho, principalmente para pessoas com deficiência intelectual, cujo quadro se constitui por entraves expressivos na funcionalidade intelectual, seguida por dificuldades exacerbadas no comportamento adaptativo, que se manifesta nas habilidades conceituais, sociais e práticas, originando-se antes dos 18 anos de idade (AAIDD, 2010, 2012; FONTES; PLETSCH; BRAUN; GLAT, 2015; REDIG, 2014, 2016 entre outros).

Quando debatemos a política de Educação Inclusiva (BRASIL, 2008, 2015, entre outras), um dos desafios é a escolarização desses jovens e adultos com deficiência, principalmente os com deficiência intelectual, no Ensino Fundamental II e Médio. Luiz e Fernandes (2018, p. 10) apontam para a existência de "poucas pesquisas que focam o tema para a pessoa com deficiência intelectual".

É preciso, portanto, ressignificar o percurso formativo desse aluno, de forma a contemplar o desenvolvimento de habilidades para uma vida independente, auxiliando no processo de transição da escola para a vida adulta. Esse período de transição para a vida pós-escola, de acordo com Carvalho (2018, p. 37), envolve "um período importante e potencialmente difícil para todos os jovens, já que têm de ser capazes de tomar decisões acerca do seu futuro". Nessa direção, o objetivo deste trabalho, com base em uma revisão sistemática da literatura específica da temática, é discutir a transição da 
escola para a vida independente, com ênfase no mundo do trabalho para jovens e adultos com deficiência intelectual, de forma a repensar como a escola pode auxiliar nesse processo.

\section{A ESCOLA COMO AGENTE FACILITADOR DA TRANSIÇÃO PARA A VIDA ADULTA}

O término da escolarização, do Ensino Médio, é compreendido como fim de uma etapa e é a marca para um novo início, que pode ser a aprovação em um curso superior, um curso profissionalizante, uma colocação no mercado de trabalho, a construção de uma família, dentre outros aspectos. Entretanto, esse é o momento em que se espera que os jovens encontrem o seu lugar na sociedade, porém, é necessário que esses, e aqui nos referimos, principalmente, aos com deficiência intelectual, tenham adquirido habilidades que auxiliem na sua inserção na sociedade e no mundo do trabalho.

A escola precisa construir metodologias inclusivas que proporcionem a esse educando com deficiência possibilidades de inserção social e laboral, juntamente com uma escolarização adequada, que englobe aspectos voltados para a aquisição de conhecimentos, desenvolvimento psicossocial e laboral. Redig (2016) expõe que as escolas, comumente, concentram-se no ensino acadêmico, não oportunizando aos jovens e adultos com deficiência intelectual, experiências que visem a capacitação para a vida adulta, independente e laboral. Entretanto, sem ações sob esse viés, as oportunidades de sucesso na conquista de trabalho para eles se tornam irrealizáveis.

Nesse contexto, a escola exerce a importante função na oferta de soluções para favorecer o desenvolvimento desse processo.

\footnotetext{
É necessário que as escolas promovam uma formação que oportunize aos seus alunos uma provisão de serviços de qualidade voltados para inclusão no mundo do trabalho e na vida social adulta, de modo geral. Nesse sentido, a preparação profissional deve ocorrer enquanto o jovem ainda esteja na escola, independentemente do espaço onde aconteça a escolarização, seja em escola comum ou em uma instituição especializada. (MASCARO, 2016, p. 11)
}

A transição para a vida adulta faz parte do cotidiano dos jovens em geral, independentemente de terem ou não deficiência. De acordo com Camarano (2006), as mudanças originárias desse processo são explicitadas por razões existentes nos ambientes escolar, profissional e familiar. Dentre outras questões, estão os problemas encontrados na inclusão profissional dos jovens e a escolarização que, ultimamente, não têm sido o bastante para garantirem a inserção desse indivíduo no mercado de trabalho. Em termos práticos, a transição se estabelece como um exercício gradativo de novos papéis 
e transformação dos papéis atuais (FÂNZERES, 2017). Contudo, para os jovens com deficiência intelectual, essa etapa, é rodeada de inseguranças. Sendo assim,

o processo de transição para a vida ativa deve ser entendido como um processo que se constrói ao longo do tempo, onde se destaca a relevância do investimento feito por cada um dos agentes sociais (escola, família, sociedade/comunidade) para o crescimento e desenvolvimento do indivíduo, seja ou não portador de deficiência (LOPES et al, 2017, p. 49)

Para Lopes (2016), a transição para a vida adulta se trata de uma parte de um longo processo e requer um empenho para o qual é importante que a pessoa seja orientada. A autora defende, também, que a transição da escola para o trabalho da pessoa com deficiência não acontece linearmente, mas precisa do comprometimento da família e do próprio sujeito, além da relação de cooperação a ser estabelecida com o setor empregatício.

Redig, Mascaro e Estef (2016, p. 06) assinalam que "para que a transição para a vida adulta e possível ingresso no mercado de trabalho de jovens com deficiência, seja menos conflituosa e estressante, é importante a elaboração de metas educacionais e um currículo flexível”. No entanto, as autoras Redig, Mascaro e Estef (2016) apontam que esse percurso precisa ser iniciado ainda na escola, por iniciativas que propiciem experiências de trabalho, habilidades laborais e sociais, além de um currículo distinto, que proporcione a personalização das ações. Elas ainda expõem que tais ações podem contribuir para a aprendizagem escolar desse público que ainda se encontra abaixo do que a escola deveria propiciar.

Portanto,

um dos papeis da escola é, além de ensinar os conteúdos curriculares tradicionais, trabalhar com os jovens com deficiência de forma a auxiliá-los a desenvolver suas potencialidades acadêmicas e profissionais e a conquistar a sua autonomia. Sabemos que isso é um desafio para as escolas, mas, por outro lado, não podemos continuar negligenciando a, mais do que comprovada, constatação de que se as práticas pedagógicas em relação às pessoas com deficiência intelectual e outras necessidades especiais não forem repensadas e ressignificadas, continuaremos promovendo a negação desses alunos como sujeitos da educação e protagonistas de suas próprias histórias (ANTUNES, 2012, p. 138).

É importante ressaltar que o desenvolvimento cognitivo de pessoas com deficiência intelectual, se altera conforme os apoios e/ou suportes recebidos e não necessariamente baseado em indicadores de inteligência. Sendo assim, a Deficiência Intelectual "deixa de ser vista como uma condição da pessoa, passando a ser vista como estado de funcionamento" (CARVALHO; FERNANDES, 2018, p. 5), isto é, fundamenta-se com base no desenvolvimento do indivíduo, as relações que são estabelecidas além dos apoios que recebe e não somente em métodos quantitativos 


\section{Apøe Ciênci@ Artigo}

apaeciencia.org.br/revista

regulados no quociente de inteligência avaliados pelos testes. Isso significa que quanto mais apoios ofertados, baseados nas habilidades e necessidades do indivíduo, maior será o progresso do sujeito.

Esses apoios, segundo o AAIDD (2010), estão divididos entre dimensões e intensidades, assim distribuídos:

a) Habilidades Intelectuais: São habilidades voltadas para o âmbito do funcionamento intelectivo, ou seja, o comportamento inteligente que, por sua vez, sofre influências de diferentes questões inerentes à individualidade. A intelectualidade expressa, nesse caso, a habilidade de compreensão do ambiente e reagir a ele de forma apropriada;

b) Comportamento Adaptativo - Habilidades Conceituais, Sociais e Práticas: Essa dimensão corresponde a um universo de habilidades desenvolvidas pelo indivíduo, no sentido de responder às demandas da vida cotidiana nas áreas física e social;

c) Saúde: O entendimento de saúde, nesse contexto, refere-se à condição de bem-estar físico, mental e social;

d) Participação: Trata da expertise social do indivíduo nos vários ambientes e cenários em que está envolvido (família, trabalho, lazer, dentre outros ambientes);

e) Contexto: Essa dimensão está relacionada com as diversas circunstâncias nas quais o indivíduo vive o seu cotidiano. É influenciado pelo ambiente no qual a pessoa está inserida, pelas atitudes e por fatores pessoais. O contexto é bastante significativo, pois, muitas vezes, é ele que determina as ações do sujeito, o que faz, com quem, onde e quando realiza, motivando, assim, as atividades e participação.

Sem o conhecimento real das potencialidades e necessidades do sujeito com deficiência intelectual, fica difícil definir os suportes que serão disponibilizados. Por isso, é fundamental que o jovem, o adulto com deficiência e a família estejam envolvidos neste processo, para que possa se estabelecer os pontos fortes e fracos e, assim, pensar nos apoios. As intensidades de apoio são assim classificadas (AAIDD, 2010):

a) Apoio intermitente: Consiste em um nível mais básico e circunstancial de apoio, ofertado de acordo com a necessidade do sujeito de forma esporádica e momentânea. Costuma ser mais presente em situações de transição de ciclos na vida da pessoa com deficiência, como por exemplo, auxiliar o sujeito na preparação para uma entrevista de emprego;

|Fed. Nac. das Apaes- Fenapaes $\mid$ Brasília/DF | v.1 | no 9

P. 44 - 56 |jan./jun.2018 | ISSN 2237-4329 
b) Apoio limitado: Trata-se do apoio solicitado por um determinado tempo para o atendimento de uma meta e/ou resolução de um problema específico por um tempo delimitado, como, por exemplo, aprender um determinado caminho (da casa para o trabalho);

c) Apoio contínuo (extensivo): Concerne ao apoio mais assíduo, com uma periodicidade regular (diário, semanal, mensal), podendo se estabelecer em longo prazo e em algum ambiente específico de convivência, além de poder se limitar a uma área determinada da vida da pessoa, como, por exemplo, o treinamento em uma nova função laboral;

d) Apoio pervasivo: é o apoio contínuo e intenso, presente em todos os ambientes e áreas da vida da pessoa. Aquele que requer esse tipo de apoio, precisa ser atendido em fatores elementares do cotidiano. Caracteriza-se pela constância e alta intensidade, como, por exemplo, pessoas com deficiência, que necessitam de apoio para a sua higiene pessoal, devido a alguma dificuldade que impeça a sua autonomia nessa esfera.

No que tange à transição, o sistema de apoios se faz importante, na medida em que corrobora e baliza as ações envolvidas no processo. Nesse caso, tanto as dimensões quanto os níveis de intensidade desses apoios, variarão de acordo com as demandas apresentadas pelo sujeito. Carvalho e Fernandes (2018) advogam que o modelo dos apoios se aloca como instrumento do paradigma da inclusão social, pois uma sociedade democrática, que reconheça a diversidade, deve providenciar todos os tipos de suportes.

Aranha (2001) expõe que o paradigma de suportes, antevê as ingerências determinantes e decisivas, tanto no decurso de desenvolvimento do indivíduo, quanto nas questões referentes ao ajustamento da realidade social. Nesse sentido, isso é parte importante do processo de transição para o trabalho, pois uma vez baseado na adequação do contexto social, pode-se garantir a todos, inclusive à pessoa com deficiência, as condições de acesso ao espaço comum da vida em comunidade e participar dos processos decisórios da sociedade.

\section{O PIT COMO INSTRUMENTO NORTEADOR NO PROCESSO DE TRANSIÇÃO}

Para possibilitar uma escolarização adequada para os alunos com deficiência intelectual é necessário pensar em práticas pedagógicas individualizadas como uma opção para oportunizar a inclusão escolar e social. A elaboração de práticas pedagógicas individualizadas atreladas e organizadas por documentos como o Plano de Ensino Individualizado (PEI) e Plano Individualizado 


\section{Apøe Ciênci@ Artigo}

de Transição (PIT) poderá ser construída de forma a possibilitar que a transição da escola para a vida independente aconteça de maneira estruturada e eficiente.

A Declaração de Salamanca (UNESCO, 1994) ressalta que o currículo para estudantes com deficiências necessita compreender programas específicos de transição, além de treinamento vocacional que proponha a eles um funcionamento autônomo e independente como cidadãos após a conclusão da escolarização. A Lei Brasileira de Inclusão da Pessoa com Deficiência, Lei 13.146/15 (BRASIL, 2015), garante: "V - adoção de medidas individualizadas e coletivas em ambientes que maximizem o desenvolvimento acadêmico e social dos estudantes com deficiência, favorecendo o acesso, a permanência, a participação e a aprendizagem em instituições de ensino”.

De acordo com a Agência Europeia para o Desenvolvimento em Necessidades Educativas Especiais (SORIANO, 2002), os aspectos da transição se estabelecem nos seguintes pressupostos:

a) Trata-se de um processo que deve ser respaldado por leis e efetivado por medidas políticas ajustadas;

b) Precisa avalizar a participação do aluno e acatar as suas escolhas pessoais, devendo ele, a sua família e os profissionais trabalharem em conjunto na formalização do PIT;

c) Necessita da implementação de um percurso formativo focado no progresso do aluno e em quaisquer mudanças que precisem ser inseridas no contexto escolar;

d) Deve se basear no envolvimento e na cooperação de todas as partes envolvidas;

e) Requer uma estreita colaboração entre escolas e mercado de trabalho, para que o aluno experimente as efetivas condições de trabalho;

f) Faz parte de um longo e complexo processo de preparação do aluno para a entrada na vida econômica e na fase adulta.

Segundo Soriano (2002), o International Labour Office afirma que a transição requer uma transformação nas relações, nas rotinas e na autoimagem das pessoas com deficiência, que precisam estabelecer metas e definirem o papel que anseiam exercer na sociedade. Afonso (2005) destaca que o crescimento social dentro de uma inclinação profissional só é possível na medida em que as instituições de ensino assumirem a função de formação e o fornecimento de oportunidades para jovens com deficiência intelectual se apropriarem do seu espaço no mercado de trabalho.

$\mathrm{Na}$ associação estabelecida entre a educação e o trabalho, é importante e necessária a existência de um planejamento de transição para a vida pós-escolar, a qual envolva a escola, a família e o próprio jovem. Desse modo, o PIT se configura como uma estratégia pedagógica para auxiliar na 
escolarização do aluno com deficiência, dialogando com os conteúdos acadêmicos, os seus interesses e o desenvolvimento de habilidades laborais. Esse documento é um instrumento norteador do trabalho do professor, que organiza a ação pedagógica e o atendimento educacional especializado, com o objetivo de auxiliar essa transição.

O PIT é, portanto, um

[...] documento que sintetiza o projeto de vida de cada aluno com NEE [necessidades educacionais especiais], para uma vida social adequada, com inclusão efetiva no meio envolvente (social, familiar ou numa instituição), que promova atividades de carácter ocupacional e, preferencialmente, o exercício de uma atividade profissional. É um processo dinâmico, a curto, médio e/ou longo prazo, com o propósito de desenvolver a capacitação e a aquisição de competências sociais necessárias à inserção familiar e comunitária. (BARBOSA; MIGUEL s/d, p.03).

O PIT não se trata de um documento descontextualizado, mas consiste em uma parte integrante do PEI (MASCARO, 2016). O PEI pode também auxiliar no planejamento e na elaboração de estratégias pedagógicas para o processo de aprendizagem escolar, habilidades sociais e habilidades importantes para a inclusão laboral (GLAT; PLETSCH, 2012; GLAT; VIANNA; REDIG,2012)

Redig (2019, p. 06) aponta que o PIT, tem por objetivo “organizar o processo de transição do estudante com deficiência da escola para a vida adulta e/ou mundo do trabalho, vida independente, sendo um dos eixos do PEI".

Soriano (2006, p. 24) expõe o PIT como

Um instrumento, uma ferramenta, sob a forma de documento, no qual é registrado o passado, o presente e o futuro desejado dos jovens. Ele deve incluir informação sobre o universo da vida do jovem: condições familiares, histórico médico, tempos livres, valores e background cultural, e ainda informação sobre a sua educação e formação.

Carvalho e Fernandes (2018) descrevem o PIT como um documento que trabalha na perspectiva da inserção de rotinas laborais, possibilitando, assim, a aquisição de conhecimentos e o cumprimento de regras, considerando os deveres sociais e laborais. O PIT, portanto, visa contribuir para a constituição de um plano de carreira, além de um projeto para a vida em todos os seus aspectos, construindo um atrelamento entre a escola e o mercado de trabalho, visando o desenvolvimento da formação para o trabalho de pessoas com deficiência, respondendo, nesse sentido, adequadamente, às necessidades e aptidões desses sujeitos, favorecendo o aspecto profissional, a autonomia pessoal e, consequentemente, trazendo melhorias na qualidade de vida.

A construção do PIT deve contar com a elaboração de atividades previamente escolhidas que abranjam a aquisição de habilidades consideradas relevantes para a futura atuação na sociedade e no 


\section{Apøe Ciênci@ Artigo}

trabalho. Logo, esse documento se torna um meio pelo qual as demandas apresentadas pelo estudante com deficiência podem ser elencadas, por estratégias de intervenção resolvidas.

Soriano (2006) pontua que o plano de transição está estritamente interligado ao PEI e deve ser preparado o mais cedo possível, antes mesmo do final do ensino fundamental. Objetiva-se terminar com a lacuna existente entre a escola e o mundo do trabalho. Um PIT proporciona um enquadramento, que tem por fim assegurar uma melhor inserção em atividades laborais, refletindo, então, em um processo dinâmico que, de acordo com Soriano (2006), envolve as características dos jovens (competências, capacidades e expectativas); as exigências do sector empregador e a revisão permanente de um plano de ação. Para esse autor, tais ações podem ser divididas em três fases:

a) Fase 1 - Informação, Observação e Orientação: Uma fase preparatória, organizada de acordo com o PEI. O objetivo é ajudar o jovem a escolher um trabalho e a encontrar um lugar de formação adequado.

b) Fase 2 - Formação e Qualificações: Essa fase tem como base, fundamentalmente, as ações desenvolvidas no decorrer do processo de formação. Tratando-se do jovem com deficiência, o objetivo é conseguir qualificações, competências e a respectiva certificação.

c) Fase 3 - Apropriação, Emprego e Acompanhamento: Essa fase coloca o enfoque nos resultados pretendidos. Em relação ao sujeito, o objetivo é conseguir e manter um trabalho, proporcionar qualidade de vida e garantir a manutenção no emprego.

Faz-se importante salientar que o PIT, como parte do PEI, deve ser organizado primeiramente pela equipe técnica da escola junto ao professor da Educação Especial, utilizando-se de informações oriundas de entrevistas com a família, com os alunos, além dos outros profissionais que trabalhem com esse estudante; relatórios, boletins e desempenho escolar, cursos realizados, competências e habilidades. Para a elaboração do PIT é imprescindível a valorização do conhecimento prévio do aluno com deficiência, suas aptidões e interesses. As suas dificuldades, embora registradas nesse documento, não recebem destaque.

Nesse sentido, Redig (2019, p. 11) destaca que três perguntas são importantes nesse processo: “1) Onde o aluno está hoje; 2) Para onde ele irá; e 3) Como o aluno chegará lá”. A autora pondera, ainda, que o acesso à essas informações, é essencial, na medida em que o PIT visa considerar "os 
pontos fortes, preferências, interesses e necessidades, apontadas no PPP [Perfil Pessoal Positivo] ${ }^{3}$ do aluno" (REDIG, 2019 p. 11). Dessarte, é possível refletir a respeito de quais estratégias podem ser utilizadas para a educação e o treinamento do sujeito, nas interfaces laborais e na vida independente.

O PIT contribui, ainda, para o autoconhecimento da pessoa com deficiência, quando essa se depara com as suas qualidades, interesses e aptidões. Dessa forma, o próprio sujeito pode criar táticas para atingir os seus objetivos, estabelecer vínculos com a comunidade e até mesmo perceber a sua deficiência.

O PIT é um plano conduzido pelo sujeito com deficiência, com a finalidade de maximizar o seu desempenho e estabelecer metas no ensino que levarão ao sucesso no momento pósescola, pois será preciso combinar experiências de vida, sociais e laborais com os conhecimentos acadêmicos. Portanto, os professores precisam aumentar as expectativas depositadas nos alunos com deficiência intelectual. (REDIG, 2019, p. 11)

Vale ressaltar que o objetivo final do PIT é conseguir com que o jovem e o adulto com deficiência intelectual possam viver de maneira mais independente e autônoma possível. Nesse caso, é fundamental uma orientação vocacional que vise despertar uma consciência de carreira. O PIT, portanto, é a peça-chave no processo de desenvolvimento do aluno com deficiência, tendo em vista que ele pode preparar esses alunos para situações cotidianas simples e complexas, como: tomada de decisões, escolhas, decisões, resolução de problemas e autogestão (CTI, 2018).

Tão quanto o PEI, o PIT também merece passar por uma avaliação ininterrupta e consciente do processo e da participação do indivíduo com deficiência, tanto na preparação quanto na implementação desse documento. Nesse caso, então, o planejamento do plano de transição precisa ser centrado no estudante e possibilitar que ele obtenha os suportes social, emocional e de aprendizagem, assessorando, dessa forma, o estudante a tomar suas decisões e informá-lo das suas opções (REDIG, 2019; U.S. DEPARTMENT OF EDUCATION, 2017).

\section{CONSIDERAÇÕES FINAIS}

O PIT exerce a função norteadora do desenvolvimento de competências para as diversas dimensões do cotidiano, como: habilidades acadêmicas, sociais, autodefensoria/autogestão, laborais, vida independente e cidadania. Essas devem ter como objetivo a saída do aluno da escola, de forma que seja capaz de se inserir socialmente na comunidade, decidir o seu percurso formativo, cursos

\footnotetext{
${ }^{3}$ O Perfil Pessoal Positivo (PPP) consiste em um inventário de habilidades, que tem como objetivo visualizar as capacidades e potencialidades do sujeito. Para maiores informações ver REDIG $(2012 ; 2014 ; 2016)$.
}

Fed. Nac. das Apaes- Fenapaes $\mid$ Brasília/DF $\mid$ v.1 | no 9

P. $44-56$

jan./jun.2018 | ISSN 2237-4329| 


\section{Apøe Ciênciø Artigo}

apaeciencia.org.br/revista

profissionalizantes e inserção no mundo do trabalho, favorecendo o seu crescimento pessoal, para que o sujeito com deficiência se torne a protagonista da sua vida. Sendo assim, conclui-se que a individualização do ensino aliada à acessibilidade pedagógica, uma equipe capacitada e com um trabalho colaborativo, possibilita que esses educandos trilhem seus caminhos nos mais diferentes níveis de ensino e vida independente (PINHEIRO; REDIG, 2019; REDIG, 2019).

Para que isso de fato ocorra, é imprescindível a criação de uma força tarefa em prol do aluno, na qual professores, tanto da turma comum quanto da Educação Especial, equipe técnica pedagógica, família, além dos demais profissionais, comunidade e o próprio aluno estejam engajados nesse processo.

\section{REFERÊNCIAS}

AFONSO C. Inclusão e mercado de trabalho - Papel da escola na transição para a vida adulta de alunos com NEE. Porto: Saber e Educar, 2005.

ANTUNES, K. C. V. História de Vida de alunos com deficiência intelectual: percurso escolar e a constituição do sujeito. 2012. f. Tese de Doutorado, Programa de Pós-Graduação em Educação, Universidade do Estado do Rio de Janeiro, Rio de Janeiro, 2012.

ARANHA, M. S. F. Paradigmas da relação da sociedade com as pessoas com deficiência. Revista do Ministério Público do Trabalho, São Paulo, ano XI, n. 21, p. 2001. Disponível em <http://www.adiron.com.br/arquivos/paradigmas.pdf> Acessado em: 19 set. 2019.

ASSOCIAÇÃO Americana de Deficiências Intelectual e do Desenvolvimento (AADID). Avaliação, Diagnóstico e Classificação. Washington, DC: AAIDD, 2010

AMERICAN Association on Intellectual and Developmental Disabilities. User's Guide to accompany the 11th edition of Intellectual Disability: Definition, Classification, and Systems of Supports. Washington, DC: AAIDD, 2012.

BARBOSA, C.; MIGUEL, J. Plano Individual de Transição. s/d. Disponível em <https://www.cfaematosinhos.eu/PIT\%20Joao\%20Miguel_Cristina\%20Barbosa_1.pdf> Acessado em: 19 set. 2019.

BRASIL. Política Nacional de Educação Especial na Perspectiva da Educação Inclusiva. 2008.

BRASIL. Lei n ${ }^{\circ}$ 13.146, de 6 de julho de 2015. Institui a Lei Brasileira de Inclusão da Pessoa com Deficiência (Estatuto da Pessoa com Deficiência). Disponível em:

<http://www.planalto.gov.br/ccivil_03/_ato2015-2018/2015/lei/113146.htm> Acessado em: 19 set. 2019.

| Fed. Nac. das Apaes- Fenapaes $\mid$ Brasília/DF | v.1 | no 9

P. 44 - 56 | jan./jun.2018|ISSN 2237-4329| 


\section{Apøe Ciênciø Artigo}

apaeciencia.org.br/revista

CAMARANO, A. A. Transição para a vida adulta ou vida adulta em transição? In: CAMARANO, A. A. (Org.) Transição para a vida adulta ou vida adulta em transição? Rio de Janeiro: Ipea, 2006.

CARVALHO, A. C. de. Plano Individual de Transição para vida adulta para pessoas com Deficiência Intelectual. 2018. f. Dissertação do Curso de Mestrado Profissional em Diversidade e Inclusão. Universidade Federal Fluminense, Niterói, RJ, 2018.

CARVALHO, A. C.; FERNANDES, E. M. Plano Individual de Transição para Vida Adulta para Pessoas com Deficiência Intelectual. In: Anais do V Colóquio Internacional Educação, Cidadania e Exclusão. Niterói, 2018.

CENTER on Transition Innovations (CTI). Lesson Four Transcript. Postsecondary Education \&amp; Training: Opening Doors to Opportunities. Virginia - EUA, 2018.

FÂNZERES, L. J. L. T. Transição para a vida adulta de alunos com necessidades educativas especiais: percursos de formação no sistema educativo Português. 2017. f. Tese (Doutorado), Programa de Pós-Graduação do Instituto de Educação da Universidade do Minho, Portugal, 2017.

FONTES, R. de S.; PLETSCH, M. D.; BRAUN, P.; GLAT, R. Estratégias pedagógicas para inclusão de alunos com Deficiência Intelectual no ensino regular. In: GLAT, R. (Org.), Educação Inclusiva: Cultura e cotidiano escolar. 2. ed. Rio de Janeiro: Sette Letras, 2015.

GLAT, R.; VIANNA, M. M.; REDIG, A. G. Plano educacional individualizado: uma estratégia a ser construída no processo de formação docente. Ciências Humanas e Sociais em Revista, v. 34, n. $1,2012$.

GLAT. R.; PLETSCH, M. D. Inclusão Escolar de alunos com necessidades educacionais especiais. 2. ed. Rio de Janeiro: EdUERJ, 2012.

LOPES, B. J. S. Programa de transição para a vida adulta de jovens com Deficiência Intelectual em ambiente universitário. 2016. f. Tese, Programa de Pós-Graduação em Educação Especial, Universidade Federal de São Carlos, São Carlos, 2016.

LOPES, M.C.S.; SILVA, A. ALVES; J. PAÇO, E. Percepções dos professores face à transição para a vida ativa de alunos com NEE. Revista de Estudios e Investigación en Psicología y Educación, Espanha, v. Extr., n. 07, 2017.

LUIZ, L. C. O.; FERNANDES, S. A transição para vida pós-escolar das pessoas com deficiência. In: Anais do VIII Congresso Brasileiro de Educação Especial. São Carlos: 2018.

MASCARO, C. Inclusão e profissionalização do aluno com Deficiência Intelectual. Curitiba: Appris, 2016.

PINHEIRO, V. C. S., REDIG, A. G. Plano Individualizado de Transição: Uma análise do documento desenvolvido pela European Agency for Development in Special Needs Education. In:

Fed. Nac. das Apaes- Fenapaes $\mid$ Brasília/DF | v.1 | no 9 | P. 44 - 56 | jan./jun.2018 |ISSN 2237-4329| 


\section{Apøe Ciênciø Artigo}

apaeciencia.org.br/revista

Anais do X Seminário Internacional As Redes Educativas e as Tecnologias. Rio de Janeiro, 2019.

REDIG, A. G. Perfil Pessoal Positivo: Estratégia para favorecer a inserção da pessoa com deficiência intelectual no mercado de trabalho. In: Anais do IV Congresso Brasileiro de Educação Especial e VII Encontro Nacional dos Pesquisadores da Educação Especial. São Carlos: UFSCar, 2012. p. 1315-1330.

REDIG, Annie Gomes. Aplicação e análise de um programa customizado para a inclusão de jovens com deficiência intelectual em atividades laborais. 2014.nf. Tese (Doutorado), Programa de Pós-Graduação em Educação, Universidade do Estado do Rio de Janeiro, 2014.

REDIG, A. G. Inserção profissional de jovens e adultos com deficiência intelectual. Curitiba: Editora Appris, 2016.

REDIG, A. G. Caminhos formativos no contexto inclusivo para estudantes com deficiência e outras condições atípicas. Revista Educação Especial, Marília - São Paulo, v. 32, p. 1-19, 2019.

REDIG, A. G., MASCARO, C. A. A. de C., ESTEF, S. Estudantes com Deficiência Intelectual: perspectivas para a vida adulta e o plano individual de transição. In: VII Congresso Brasileiro de Educação Especial. São Carlos: 2016.

SORIANO, V. Transição da Escola para o Emprego. Principais problemas, questões e opções enfrentadas pelos alunos com necessidades educativas especiais em 16 países Europeus. European Agency for Development in Special Needs Education, 2002.

SORIANO, V. Planos Individuais de Transição: apoiar a transição da escola para o emprego. European Agency for Development in Special Needs Education, 2006.

U. S. DEPARTMENT OF EDUCATION (Department). Office of Special Education and Rehabilitative Services. A Transition Guide to Postsecondary Education and Employment for Students and Youth with Disabilities. Washington, D.C., EUA, 2017.

UNESCO. Declaração de Salamanca e linha de ação sobre necessidades educativas especiais. Brasília: CORDE, 1994. 